\title{
Quality of life and survival survey of cancer cachexia in advanced non-small cell lung cancer patients-Japan nutrition and QOL survey in patients with advanced non-small cell lung cancer study
}

\author{
Koichi Takayama ${ }^{1,2} \cdot$ Shinji Atagi $^{3}$ - Fumio Imamura ${ }^{4}$ Hiroshi Tanaka ${ }^{5}$ Koichi Minato ${ }^{6}$. \\ Toshiyuki Harada ${ }^{7}$ - Nobuyuki Katakami ${ }^{8}$. Takuma Yokoyama ${ }^{9}$ Kozo Yoshimori ${ }^{10}$. \\ Yuichi Takiguchi $^{11}$ - Osamu Hataji ${ }^{12}$ - Yuichiro Takeda ${ }^{13} \cdot$ Keisuke Aoe $^{14}$. Young Hak Kim ${ }^{15}$. \\ Soichiro Yokota ${ }^{16}$. Hiroshi Tabeta ${ }^{17} \cdot$ Keisuke Tomii $^{18}$ - Yasuo Ohashi ${ }^{19} \cdot$ Kenji Eguchi $^{20}$. \\ Koshiro Watanabe ${ }^{21}$
}

Received: 24 September 2015 / Accepted: 7 March 2016 / Published online: 22 March 2016

(C) The Author(s) 2016. This article is published with open access at Springerlink.com

\begin{abstract}
Purpose Although cancer cachexia is mainly characterized by persistent loss of body weight (BW), usually in response to a malignancy, the pathophysiology of cachexia remains unresolved. To elucidate the relationship between the loss of BW and other related clinical factors, we conducted a nationwide, multi-institutional, prospective, observational study in patients with advanced non-small cell lung cancer (NSCLC).
\end{abstract}

Electronic supplementary material The online version of this article (doi:10.1007/s00520-016-3156-8) contains supplementary material, which is available to authorized users.

Koichi Takayama

takayama@koto.kpu-m.ac.jp

1 Department of Pulmonary Medicine, Kyoto Prefectural University of Medicine, Kyoto, Japan

2 Department of Respirology, Kyoto Prefectural University of Medicine, 465 Kajii-cho, Kamigyo-ku, Kyoto 602-8566, Japan

3 Department of Thoracic Oncology, National Hospital Organization Kinki-chuo Chest Medical Center, Sakai, Japan

4 Department of Thoracic Oncology, Osaka Medical Center for Cancer and Cardiovascular Diseases, Osaka, Japan

5 Department of Internal Medicine, Niigata Cancer Center Hospital, Niigata, Japan

6 Department of Respiratory Medicine, Gunma Prefectural Cancer Center, Ota, Japan

7 Center for Respiratory Diseases, Japan Community Health care Organization, Hokkaido Hospital, Sapporo, Japan
Methods Treatment-naïve stage IV NSCLC patients with an Eastern Cooperative Oncology Group performance status (PS) of 0-2 were eligible. BW, handgrip strength (HGS), quality of life (QOL), Karnofsky Performance Scale (KPS), biochemical parameters, and survival were evaluated at baseline and every 4 weeks for 1 year. The relationship between BW loss and other factors was examined by linear regression analysis. Estimated survival curves were drawn by the Kaplan-
8 Division of Integrated Oncology, Institute of Biomedical Research and Innovation, Kobe, Japan

9 Department of Respiratory Medicine, Kyorin University Hospital, Mitaka, Japan

10 Department of Clinical Oncology, Japan Anti-Tuberculosis Association, Fukujuji Hospital, Kiyose, Japan

11 Department of Medical Oncology, Graduate School of Medicine, Chiba University, Chiba, Japan

12 Respiratory Center, Matsusaka Municipal Hospital, Matsusaka, Japan

13 Department of Respiratory Medicine, National Center for Global Health and Medicine, Tokyo, Japan

14 Department of Medical Oncology, National Hospital Organization Yamaguchi-Ube Medical Center, Ube, Japan

15 Department of Respiratory Medicine, Graduate School of Medicine, Kyoto University, Kyoto, Japan

16 Department of Respiratory Medicine, National Hospital Organization Toneyama National Hospital, Toyonaka, Japan 
Meier method and applied by the log-rank test. Clinical factors associated with cancer cachexia were identified through principal component analysis. The generalized estimating equation approach was used to analyze the deterioration of QOL resulting from the progression of cachexia.

Results A total of 406 patients were analyzed. BW loss was significantly associated with worsening of QOL, HGS, KPS, and biochemical parameters. The incidence of BW loss was observed throughout the study period. Overall survival was significantly shorter in patients as BW loss progressed. BW loss, decrease in HGS, anorexia, and fatigue were identified as core factors of cachexia that contributed to the deterioration of QOL.

Conclusion BW loss most likely deteriorated QOL and shortened survival in patients with advanced NSCLC and should be closely monitored.

Keywords Non-small cell lung cancer · Body weight loss · Handgrip strength · Karnofsky Performance Scale · Quality of life

\section{Introduction}

Body weight (BW) loss is a common clinical manifestation in cancer patients, especially in those with advanced stage cancer. Cancer-related BW loss is usually associated with reduced physical function mainly owing to muscle wasting. Consequently, cancer-related BW loss can lead to worsening of patients' general condition and deterioration of performance status (PS) and quality of life (QOL) [1,2]. Moreover, BW loss is a well-known prognostic factor in various malignant diseases and a prominent feature of cancer cachexia. Evans et al. defined cachexia as a metabolic syndrome associated with underlying illness, characterized by the loss of muscle with or without the loss of fat mass [3]. Anorexia, inflammation, insulin resistance, hypogonadism, anemia, and others all form the complex web that underlies the development of cachexia. To maintain an acceptable condition and QOL during cancer care, it is necessary to address the clinical issues surrounding cancer cachexia. However, details of the onset mechanism of cancer cachexia remain unknown.

\footnotetext{
17 Department of Pulmonary Medicine, Funabashi Municipal Medical Center, Funabashi, Japan

18 Department of Respiratory Medicine, Kobe City Medical Center General Hospital, Kobe, Japan

19 Department of Integrated Science and Engineering for Sustainable Society, Chuo University, Tokyo, Japan

20 Health Science on Supportive Medicine for Intractable Diseases, Teikyo University School of Medicine, Tokyo, Japan

21 Thoracic Oncology Research Group, Yokohama, Japan
}

Lung cancer is a leading cause of cancer-related deaths in Japan. In 2012, 71,518 lung cancer patients died [4]. In lung cancer patients, BW loss is a common occurrence and a matter of serious concern. Over the past 30 years, the frequency of BW loss in lung cancer patients has consistently exceeded that of other malignant diseases $[5,6]$. To our knowledge, however, no large prospective study focusing on cancer-related BW loss has been reported in the field of lung cancer. In addition, BW loss has been known to deteriorate PS, QOL, and the prognosis of the patients, but it is unknown whether this is still applicable today when therapies for non-small cell lung cancer (NSCLC) have advanced significantly because of the development of new treatments such as molecularly targeted drugs. Therefore, we conducted a nationwide, multi-institutional, prospective, observational study to investigate the relationship between BW loss and related clinical parameters in patients with advanced non-small cell lung cancer (NSCLC). This study was conducted as the Japan Nutrition and QOL survey in patients with advanced NSCLC study (JNUQ-LC Study, TORG0912). It is hoped that this study will be helpful in further understanding cancer cachexia and optimizing the use of forthcoming anti-cachexia agents.

\section{Patients and methods}

\section{Study design}

The aim of this study was to observe the clinical course of advanced NSCLC focusing on BW and to elucidate the relationship between changes in $\mathrm{BW}$ and related clinical factors. The study was conducted at 75 facilities throughout Japan. All patients signed an informed consent. The study was approved by the institutional review boards of all participating facilities and was conducted in compliance with institutional review board regulations. This study is registered with University Hospital Medical Information Network-Clinical Trial Registry (UMIN-CTR) identification number: UMIN000003630.

\section{Population}

Patients with pathologically confirmed stage IV NSCLC, according to the 7th edition of the UICC TNM classification, were eligible for enrollment. Other eligibility criteria included age 20 years or older at the time of consent, being chemotherapy-naïve, and having an Eastern Cooperative Oncology Group (ECOG) performance status of 0-2 (on a scale of 05 , with 0 indicating that the patient is asymptomatic and higher numbers indicating increasing disability). Exclusion criteria included having surgery planned during the study period; inability to complete QOL questionnaires or perform a handgrip strength (HGS) test; and the presence of severe 
digestive diseases impeding oral ingestion, synchronous multiple primary cancers, uncontrolled severe diabetes mellitus, and uncontrolled psychiatric disorders.

\section{Measurements of clinical parameters}

All clinical parameters were evaluated within 2 weeks before treatment as baseline data and every 4 weeks for 1 year after the start of treatment. Patients' BW was measured by weight scale (HBF-362, Omron Corporation, Kyoto, Japan) provided by the registration center. HGS was measured by grip dynamometer (Jamar Hydraulic Dynamometer, model \#MG4005NC, Patterson Medical Holdings, Inc., Bolingbrook, IL) provided by the registration center. The HGS test was repeated three times each in the dominant and non-dominant hand, according to the procedure manual. QOL was scored by two screening questionnaires: the Japanese version of the M.D. Anderson Symptom Inventory (MDASI-J) [7] and the QOL questionnaire for cancer patients treated with anticancer drugs (QOL-ACD) [8]. The general condition of patients was evaluated using the Karnofksy Performance Scale (KPS). Biochemical assays, including white blood cell count and hemoglobin in peripheral blood, total protein, albumin, triglycerides, calcium, C-reactive protein (CRP), and insulin-like growth factor-1 (IGF-1) in serum, were also performed. Interleukin-6 (IL-6), acyl-ghrelin, des-acyl-ghrelin, leptin, and parathyroid hormone-related protein $(\mathrm{PTHr})$ were sampled and analyzed as optional parameters.

The modified Glasgow prognostic score (mGPS), an independent inflammation-based prognostic score of nutritional status in lung cancer patients, was tabulated. The mGPS was constructed using CRP and albumin as follows: patients with both an elevated CRP $(\geq 10 \mathrm{mg} / \mathrm{L})$ and low albumin $(<35 \mathrm{~g} / \mathrm{L})$ were allocated a score of 2 ; patients in whom only CRP was elevated $(\geq 10 \mathrm{mg} / \mathrm{L})$ were allocated a score of 1 ; and patients with a normal CRP were allocated a score of 0 [9].

\section{Statistical analysis}

We hypothesized that cancer cachexia characterized by BW loss is tightly associated with QOL worsening. The relationship between various clinical parameters and BW change or QOL change from baseline was determined by structural equation modeling. McDonald's fit index was used to assess the fit of the structural equation model to data. This study was designed to have $80 \%$ power to select the model using the goodness-of-fit test and McDonald's fit index $>0.95$ with a type I error rate of 0.05 (two-sided) [10]. Taking into account a dropout rate of $30 \%$, the sample size was determined to be 440 .

To explore the change in BW loss, we compared the incidence of $\geq 5 \%$ loss of BW in some time periods with patients' baseline BW using an incidence rate ratio (95\% confidence limit). The relationship between BW loss and other parameters was analyzed by simple and multiple linear regression analyses. The relative risk (95\% confidence interval [CI]) of BW loss $(\geq 5 \%)$ in patients experiencing progressive disease (PD) versus patients not experiencing PD, within 8 weeks after registration, and Fisher's exact test were calculated to evaluate whether early PD would affect BW loss. The association between specific therapy (treatment in the first 4 weeks after registration) and $\mathrm{BW}$ loss was evaluated in the same manner as early PD. To examine the relation between BW loss and cancer nutritional therapy, we compared the proportion of patients taking cancer nutritional therapy with BW loss levels and calculated the relative risk using a $95 \% \mathrm{CI}$. The correlation between BW loss (and/or mGPS) and overall survival time was evaluated using standard survival analysis procedures. Estimated Kaplan-Meier plots and the log-rank test were applied to assess the statistical difference. Additionally, the Cox proportional hazards model was used to estimate the hazard ratios $(95 \% \mathrm{CI})$. Survival time was calculated from the date of registration to the date of death from any cause. Clinical factors associated with cancer cachexia were extracted through principal component analysis. We conducted this principal component analysis using the definition of cancer cachexia by Evans et al. for convenience because we needed to define it [3]. The criteria included BW loss of at least $5 \%$ over 6 months or less in the presence of an underlying illness plus three of the following: decreased muscle strength $(\geq 5 \%$ decrease in HGS for both the dominant and non-dominant hands), fatigue ( $\geq$ grade 1 of CTCAE), anorexia ( $\geq$ grade 1 of CTCAE), and abnormal biochemistry (CRP $>5.0 \mathrm{mg} / \mathrm{L}$, hemoglobin $<12 \mathrm{~g} / \mathrm{dL}$, and serum albumin $<3.2 \mathrm{~g} / \mathrm{dL}$ ). Low fatfree mass index was not included in these criteria because body composition was not investigated in the study. The relationship between these extracted factors and QOL deterioration was examined by a generalized estimating equation (GEE).

All missing values were not imputed. $p<0.05$ was considered statistically significant. All statistical analyses were performed using SAS software (version 9.2 or 9.3, SAS Institute).

\section{Results}

\section{Patient demographics}

Seventy-five facilities participated in the study. From February to July 2010, 466 patients were registered. Sixty patients were excluded due to the lack of BW data. The remaining 406 patients ( 280 male, 126 female) were evaluable and analyzed. Patient demographics are shown in Table 1. The median age was 67 years (range, 33-87). 
The median BW was $56.5 \mathrm{~kg}$ (range, 31.9-92.7), and the mean body mass index was 21.79 . The proportion of subjects with $>5 \%$ BW loss observed within 6 months before registration in the study was $7.4 \%$; however, optional data were missing in $46.6 \%$ of the registered subjects. Patients' PS ranged from 0 to 2: 0 $(n=159), 1(n=209)$, and $2(n=38)$. The mGPS was $0(n=373), 1(n=1)$, and $2(n=27)$. These data showed that the general status of most of the study subjects was favorable.

\section{Incidence of BW loss by observational period}

The 52-week observational period was divided into four periods: first, 4 to 12 weeks; second, 16 to 24 weeks; third, 28 to 36 week; fourth, 40 to 52 weeks. The incidence rates of $\geq 5 \%$ BW loss were similar in each period. The incidence rate ratios of $\geq 5 \%$ BW loss (vs first) were second $=1.127$, third $=1.059$, and fourth $=1.158$ (Table 2).

\section{Relationship between BW loss and QOL}

Four hundred six patients were divided into four groups equally according to the percentage of BW loss from baseline: first quartile, BW loss $\leq 2.3 \%$; second quartile, BW loss $2.3 \%$ $\leq 6.1 \%$; third quartile, BW loss $6.1 \% \leq 10.9 \%$; and fourth quartile, BW loss $>10.9 \%$. QOL was evaluated and scored using the MDASI-J questionnaire. Figure 1 shows the

Table 1 Patient demographics

\begin{tabular}{lll}
\hline Gender & Male & $280(69.0 \%)$ \\
\hline \multirow{2}{*}{ Age } & Female & $126(31.0 \%)$ \\
& Mean \pm SD & $67.0 \pm 10.1$ \\
Height (cm) & Median (range) & $67(33-87)$ \\
& Mean \pm SD & $161.5 \pm 8.9$ \\
Body weight (kg) & Median (range) & $162.0(134.6-185.0)$ \\
& Mean \pm SD & $56.9 \pm 10.1$ \\
BMI & Median (range) & $56.5(31.9-92.7)$ \\
Performance status & Mean \pm SD & $21.79 \pm 3.12$ \\
& 0 & $159(39.2 \%)$ \\
Body weight loss & 1 & $209(51.5 \%)$ \\
Within 6 months & 2 & $38(9.4 \%)$ \\
Before registration & $>5 \%$ & $187(46.1 \%)$ \\
Modified Glasgow & Unmeasured & $30(7.4 \%)$ \\
Prognostic score & 1 & $373(91.9 \%)$ \\
& 2 & $1(0.2 \%)$ \\
& Unmeasured & $27(6.7 \%)$ \\
& $5(1.2 \%)$ \\
\hline
\end{tabular}

$B M I$ body mass index distribution of a QOL score change from baseline in each group. Greater BW loss was associated with worsened QOL. The regression coefficient was $-0.0291(P=0.0002)$, and the relationship between BW loss and QOL worsening was statistically significant. MDASI-J includes four subscales that reflect symptom severity, interference of daily activity, fatigue, and appetite loss. A similar analysis was performed on the relationship between BW loss and each subscale. Regression coefficients were -0.5268 in symptom severity $(P=0.0007),-0.3795$ in interference of daily activity $(P=0.0003),-0.2596$ in fatigue $(P=0.0077)$, and -0.2652 in appetite loss $(P=0.0038)$. BW loss was clearly correlated with the worsening of clinical factors in each subscale.

Likewise, the relationship between BW loss and QOL evaluated by the QOL-ACD questionnaire was investigated. As shown in Supplementary Fig. 1, a significant relationship was observed with a regression coefficient of $0.0631(P<0.0001)$. Regression coefficients in four subscales were 0.1234 in daily activity $(P<0.0001), 0.2238$ in physical condition $(P<0.0001), 0.1700$ in psychological condition $(P=0.0003)$, and 0.1509 in sociality $(P=0.0017)$. In the face scale of QOL-ACD, used to evaluate general QOL, a strong relationship was observed with a regression coefficient of $0.8457(P<0.0001)$.

\section{Relationship between BW loss and performance status}

The distribution of KPS change in each group is shown in Supplementary Fig. 2. The regression coefficient was 0.0363 $(P<0.0001)$. Greater BW loss correlated with worse performance status.

\section{Relationship between BW loss and handgrip strength}

The distribution of HGS change in the non-dominant hand in each BW loss group is shown in Supplementary Fig. 3. The regression coefficient was $0.4363(P<0.0001)$ in the non-dominant hand and $0.3915(P<0.0001)$ in the dominant hand. Patients with more BW loss showed greater decreases in HGS, especially in the non-dominant hand. Similarly, the relationship between HGS change and QOL score change or KPS change was analyzed. The decrease in HGS in the non-dominant hand was clearly related to significant worsening of QOL or KPS (data not shown).

\section{Relationship between BW loss and biochemical parameters}

BW loss correlated with several biochemical parameters. The regression coefficients were 0.1312 in lymphocyte count $(P<0.0001), 0.7364$ in hemoglobin $(P<0.0001), 2.2637$ in serum albumin $(P<0.0001)$, 
Table 2 Incidence rate of weight loss $(\geq 5 \%)$ in observational periods in patients with advanced non-small cell lung cancer

\begin{tabular}{lllll}
\hline & \multicolumn{2}{l}{ Observational periods } & \\
\cline { 2 - 5 } & First & Second & Third & Fourth $^{\mathrm{a}}$ \\
\hline Range of week & $4-12$ & $16-24$ & $28-36$ & $40-52$ \\
Total number of observation & 1112 & 865 & 704 & 713 \\
Weight loss ( $\geq 5 \%), n$ & 276 & 242 & 185 & 205 \\
Incidence rate & 0.248 & 0.280 & 0.263 & 0.288 \\
$95 \%$ CL (lower, upper) & $(0.221,0.279)$ & $(0.247,0.317)$ & $(0.228,0.304)$ & $(0.251,0.330)$ \\
Incidence rate ratio & & & & \\
$($ vs first ) & 1 & 1.127 & 1.059 & 1.158 \\
$95 \%$ CL (lower, upper) & - & $(0.948,1.340)$ & $(0.879,1.275)$ & $(0.967,1.388)$ \\
$P$ value & - & 0.1740 & 0.5479 & 0.1108 \\
\hline
\end{tabular}

The 52-week observational period was divided into four periods: first, 4 to 12 weeks; second, 16 to 24 weeks; third, 28 to 36 weeks; fourth, 40 to 52 weeks

$C L$ confidence limit

${ }^{\text {a }}$ Four observation periods in fourth period only; all others had three observation periods
4.6048 in serum triglycerides $(P=0.0011),-1.2260$ in CRP $(P=0.0016), 0.0218$ in IGF-1 $(P=0.0023)$, and 10.8947 in leptin $(P=0.00358)$. There was no correlation between BW loss and other parameters (IL-6, acylghrelin, des-acyl-ghrelin, and PTHrP), partly owing to the small sample number.

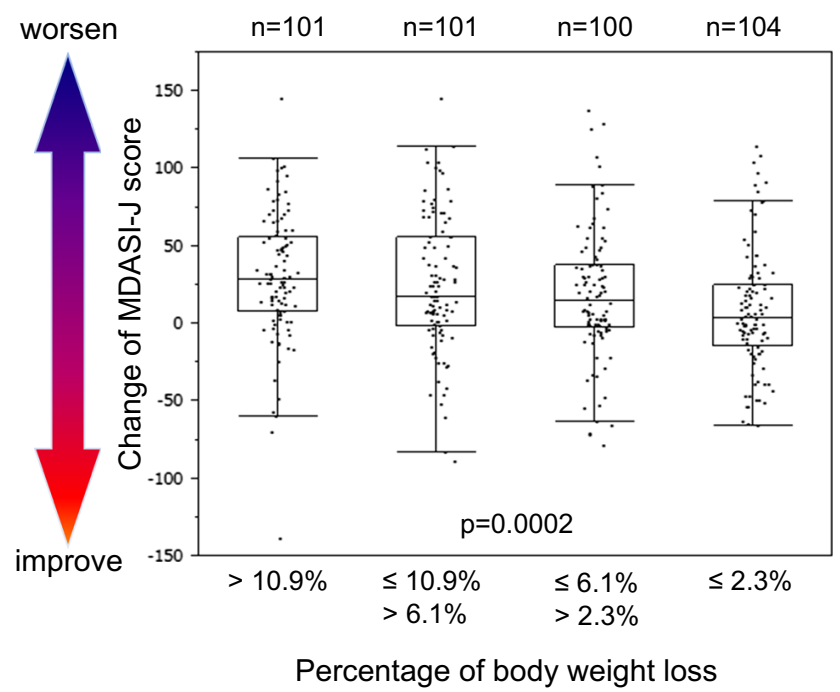

Fig. 1 Relationship between body weight loss and change of MDASI-J score. Increase in score change indicates worsening of quality of life (QOL). Four hundred six patients were divided into four groups equally according to percentage of body weight (BW) loss from baseline. First quartile includes patients with BW loss $\leq 2.3 \%$; second quartile, BW loss $2.3 \% \leq 6.1 \%$; third quartile, BW loss $6.1 \% \leq 10.9 \%$; and fourth quartile, BW loss $>10.9 \%$. QOL was evaluated and scored using MDASI-J questionnaire. Regression coefficient was $-0.0291(P=0.0002)$ with linear regression analysis. MDASI-J, the Japanese version of the M.D. Anderson Symptom Inventory

\section{Investigation of factors possibly affecting BW loss}

Factors possibly associated with BW loss were investigated. These included PD, anticancer therapy, and nutritional therapy (intake of food high in nutrition and high-calorie infusion). BW loss occurred more frequently in patients with PD than in patients with non-PD (relative risk, 1.30; $95 \% \mathrm{CI}, 1.08$ $1.58 ; P=0.0254$ ). No difference was observed on the influence of BW loss among therapies (platinum-based, non-platinum-based, or molecular targeted therapy) or no therapy. Differences were observed between the molecular-targeted therapy group and the treatment-naïve group. The impact of nutritional therapy on BW loss was not confirmed; however, the number of patients who used the therapy was too small for evaluation. These data suggest that PD may affect the onset of BW loss.

\section{Overall survival}

The median overall survival (OS) in all patients $(N=384)$ was 1.15 years, with a median follow-up of 1.06 years. The comparison of OS in four quartiles by BW loss revealed statistically significant shorter OS as BW loss increased by the logrank test (first vs third: $P=0.0011$; first vs fourth: $P<0.0001$ ) (Fig. 2).

We also analyzed the effect of BW loss before enrollment. Approximately $7.4 \%$ of all patients experienced BW loss of $5 \%$ or more 6 months before registration; however, there was no statistical difference in overall survival time between patients with or without prior BW loss $(P=0.1524)$. The OS was analyzed using Cox proportional hazards model with BW loss and mGPS as covariates. Greater BW loss or a higher mGPS score (score 0 vs 1 and 2) correlated with a 
Fig. 2 Overall survival curves according to the percentage of body weight loss

The overall survival curve was drawn using the Kaplan-Meier method: first quartile, body weight (BW) loss $\leq 2.3 \%$; second quartile, BW loss $2.3 \% \leq 6.1 \%$; third quartile, BW loss $6.1 \%$ $\leq 10.9 \%$; and fourth quartile, BW loss $>10.9 \%$. $Q$ quartile, $H R$ hazard ratio

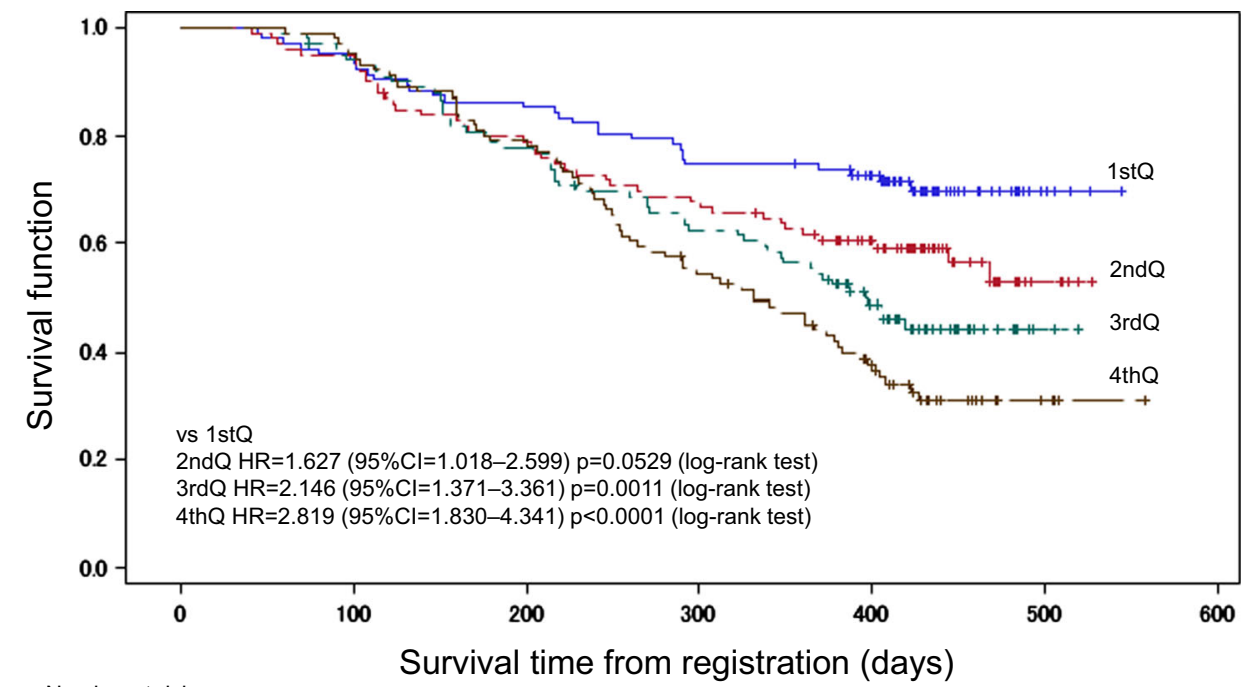

Number at risk

$\begin{array}{rlllllll}\text { 1stQ } & 103 & 97 & 88 & 77 & 66 & 5 & 0 \\ \text { 2ndQ } & 100 & 95 & 78 & 67 & 47 & 5 & 0 \\ \text { 3rdQ } & 100 & 93 & 77 & 61 & 39 & 2 & 0 \\ \text { 4thQ } & 101 & 96 & 80 & 54 & 33 & 4 & 0\end{array}$

1 stQ, body weight (BW) loss $\leq 2.3 \%$; 2ndQ, BW loss $2.3 \% \leq 6.1 \%$; 3rdQ, BW loss $6.1 \% \leq 10.9 \%$; 4 thQ, BW loss $>10.9 \%$ shorter OS. Both BW loss and mGPS were considered independent prognostic factors (Supplementary Table 1).

\section{The principal component analysis of cancer cachexia}

We listed various factors representing cancer cachexia and investigated their strength in relation to cachexia by analyzing the core components (Table 3). Four factors were found to have a high degree of impact in cancer cachexia: BW loss, decrease in HGS, anorexia, and fatigue. The degree of impact of these factors was as follows: BW loss, 0.654; decrease in HGS, 0.592; anorexia, 0.825; and fatigue, 0.749. Statistical analysis of time-course data using GEE was performed to explore the degree of progression of cancer cachexia that contributed to changes in QOL. Progression of these four factors contributed strongly to the deterioration of QOL $(P<0.01)$.

\section{Discussion}

This prospective study demonstrated that BW loss in advanced NSCLC patients correlated with worsening of QOL and KPS and a decrease in HGS. QOL was evaluated using two questionnaires: MDASI-J and QOLACD. MDASI-J was used to assess multiple symptoms experienced by cancer patients and their interference with patients' activities of daily living. The QOL-ACD focuses on patients treated with anticancer drugs and evaluates not only their physical status but also their psychological status and ability to engage in social activities. Both QOL evaluation methods demonstrated that BW loss was strongly associated with worsening of QOL. Biochemical assays suggest that systemic inflammation, malnutrition, and anemia are cofactors in involuntary weight loss. These poor general conditions contribute markedly to patients' decreased QOL, as reported previously. Furthermore, the greater the BW loss, the shorter the OS became; BW loss is a prognostic factor, independent of mGPS. Thus, BW loss in cancer

Table 3 Principal component analysis of cancer cachexia

\begin{tabular}{ll}
\hline Symptoms comprising the factor $^{\mathrm{a}}$ & Degree of impact on the factors \\
\hline Body weight loss & 0.654 \\
Decrease in handgrip strength & 0.592 \\
Fluid retention & 0.178 \\
Gastrointestinal tract disturbance & 0.420 \\
Anorexia & 0.825 \\
Motor function disorder & 0.124 \\
Pain & 0.525 \\
Fatigue & 0.749 \\
Respiratory embarrassment & 0.116 \\
Steroid administration & 0.002 \\
Parenteral hyperalimentation & 0.458
\end{tabular}

${ }^{a}$ Evaluated at date of weeks closest to when the maximum change in quality of life was observed

${ }^{\mathrm{b}}$ Figures closer to 1.000 indicate a stronger impact 
patients must be carefully monitored throughout the clinical course of treatment.

Fearon et al. recently participated in a formal consensus process and proposed a new definition of cachexia [11]. The international consensus defined cancer cachexia as a multifactorial syndrome identified by the "ongoing loss of skeletal muscle mass (with or without loss of fat mass) that cannot be fully reversed by conventional nutritional support and leads to progressive functional impairment." The diagnostic criterion agreed upon for cachexia was weight loss greater than $5 \%$ or weight loss greater than $2 \%$ in individuals already showing depletion according to current bodyweight and height (BMI $<20 \mathrm{~kg} / \mathrm{m}^{2}$ or sarcopenia) [11]. It is noteworthy that this definition took into consideration the possibility of having a patient who was relatively slim before developing cancer. Since body size between Caucasians and Asians varies (Asians being generally slimmer and smaller), the criterion should be validated before it is applied to Asian patients.

From our observations during this study, we found that BW loss occurred early after the initiation of cancer treatment and was sustained consistently throughout the 52-week observational period. The influence of anticancer therapy and nutritional therapy on BW loss was limited; however, the onset of BW loss pointed to $\mathrm{PD}$.

These results suggest the importance of recognizing BW loss in advanced NSCLC patients and intervening quickly and appropriately. The importance of initiating treatment for BW loss early is supported by a report of early palliative care for NSCLC patients, which resulted in longer patient survival [12, 13]. Fearon et al. categorized cancer cachexia into three stages: precachexia, cachexia, and refractory cachexia [11]. Because refractory cachexia is clinically unresponsive to anticancer therapy, it is important to recognize cachexia as soon as possible by monitoring BW loss, at least before it progresses to the refractory stage. In addition, we should pay more attention to BW loss because our findings have shown that the disadvantages associated with $\mathrm{BW}$ loss are still the same despite the progress in NSCLC therapies. However, wider awareness of the importance of BW loss through this study could improve care because BW can be easily measured anywhere.

As reported previously, BW loss may be associated with incompletion of chemotherapy courses and treatment-related toxicities $[14,15]$. In this study, we also analyzed the effect of BW loss prior to enrollment; however, there was no statistical difference in OS time between patients with or without prior BW loss. Although this interpretation is limited because of the small sample size, BW loss that occurred before or after systemic chemotherapy seemed to affect survival differently.
We also focused on other symptoms accompanied by BW loss and identified them by analyzing the principal components (Table 3). Our observation confirmed that cancer cachexia is characterized by anorexia, fatigue, and loss of muscle strength, as well as BW loss. These four factors are linked tightly to each other and are considered core factors in cachexia. Progression of these factors was strongly associated with worsening of QOL. This study result demonstrates that BW loss leads to decrease in clinical parameters such as QOL, KPS, and HGS. We found that the BW loss is one of the core factors of cancer cachexia, which is consistent with the criteria proposed by Fearon or Evans that implicate BW loss as a key factor in cancer cachexia [3, 11].

In patients treated with systemic chemotherapy, however, it is difficult to distinguish toxicities associated with cancer chemotherapy from cachexia-related symptoms. Since patients were treated with chemotherapy during this study, BW loss or QOL worsening was due in part to the adverse effects of treatment and is thus a limitation of this study. When symptoms possibly associated with systemic chemotherapy, such as anorexia and/or fatigue, last longer than expected, cachexia should be suspected.

Based on the results of this study, we identified that there were correlations between BW loss and the deterioration of other clinical factors such as QOL. However, we did not quantify how BW loss contributed to the changes in these factors. If these quantifications could be identified, the prophylaxis against BW loss by some treatments can contribute the deterioration of such factors. A GEE or a repeated measure ANOVA may be appropriate to solve those problems.

Cachexia is an important clinical target. Until recently, there have been attempts at therapeutic approaches to cachexia through nutritional support, rehabilitation/ exercise [16], corticosteroid administration, and herbal medicine for cancer patients, but the effectiveness of these treatments has been limited. The development of anti-cachexia drugs, such as enobosarm and anamorelin, however, offers promise. These drugs have been shown to increase total lean body mass and/or improve anorexia/cachexia symptoms [17, 18].

Continued clinical research of cachexia is essential for improving patients' tolerance to cancer therapies and QOL. The goal should be to develop preventive measures and treatment for cachexia based on a more complete understanding of the underlying pathophysiology of the syndrome.

Acknowledgments The authors thank all participating sites, investigators, and staff in the Thoracic Oncology Research Group (TORG) for their support with the clinical study. The authors are fully responsible for the content for this manuscript. 
Compliance with ethical standards The study was approved by the institutional review boards of all participating facilities and was conducted in compliance with institutional review board regulations. This study is registered with University Hospital Medical Information NetworkClinical Trial Registry (UMIN-CTR) identification number: UMIN000003630.

Conflict of interest Koichi Takayama received honoraria from Chugai Pharmaceutical, Eli Lilly Japan, Pfizer Japan, and AstraZeneca; advisory fees from Kyushu Research Support Center Kyushu; and research funds from Chugai Pharmaceutical, Eli Lilly Japan, Kyowa Hakko Kirin, and Pfizer Japan. Shinji Atagi received honoraria from Eli Lilly Japan, Chugai Pharmaceutical, Taiho Pharmaceutical, and Boehringer Ingelheim Japan. Koichi Minato's institution received research funds from Ono Pharmaceutical. Yuichi Takiguchi received honoraria from AstraZeneca, Chugai Pharmaceutical, Sanofi, and Ono Pharmaceutical and research funds from AstraZeneca, Chugai Pharmaceutical, and Sanofi. Osamu Hataji received honoraria from Novartis Pharma and research funds from Novartis Pharma, GlaxoSmithKline, Kyorin Pharmaceutical, and Boehringer Ingelheim Japan. Yasuo Ohashi received personal fees as executive salaries from Statcom; honoraria from Daiichi Sankyo, Chugai Pharmaceutical, Shionogi, EPS, Dentsu Sudler \& Hennessey, and DNP Media Create; and grants from Astellas Pharma, Takeda Pharmaceutical, Kyowa Hakko Kirin, and Kowa Pharmaceutical. Kenji Eguchi's institution is endowed research unit by Ono Pharmaceutical. All remaining authors have declared no conflicts of interest.

Funding This study was supported by Ono Pharmaceutical Co., Ltd. and was conducted by the Thoracic Oncology Research Group (JNUQLC study, TORG0912). Decisions regarding the study plan, implementation, and publication were made by the group representative, the coordinating investigator, and the investigators involved in the preparation of the protocol.

Open Access This article is distributed under the terms of the Creative Commons Attribution-NonCommercial 4.0 International License (http:// creativecommons.org/licenses/by-nc/4.0/), which permits any noncommercial use, distribution, and reproduction in any medium, provided you give appropriate credit to the original author(s) and the source, provide a link to the Creative Commons license, and indicate if changes were made.

\section{References}

1. O'Gorman P, McMillan DC, McArdle CS (1999) Longitudinal study of weight, appetite, performance status, and inflammation in advanced gastrointestinal cancer. Nutr Cancer 35:127-129

2. O'Gorman P, McMillan DC, McArdle CS (2000) Prognostic factors in advanced gastrointestinal cancer patients with weight loss. Nutr Cancer 37:36-40
3. Evans WJ, Morley JE, Argilés J, et al. (2008) Cachexia: a new definition. Clin Nutr 27:793-799

4. Japan Cancer Statistics (2012) from Center for Cancer Control and Information Service, National Cancer Center. http://ganjoho.jp/ professional/index.html. Accessed 22 July 2015

5. DeWys WD, Begg C, Lavin PT, et al. (1980) Prognostic effect of weight loss prior to chemotherapy in cancer patients. Am J Med 69: 491-497

6. Ross PJ, Ashley S, Norton A, et al. (2004) Do patients with weight loss have a worse outcome when undergoing chemotherapy for lung cancers? Br J Cancer 90:1905-1911

7. Okuyama T, Wang XS, Akechi T, et al. (2003) Japanese version of the MD Anderson Symptom Inventory: a validation study. J Pain Symptom Manag 26:1093-1104

8. Kurihara M, Shimizu H, Tsuboi K, et al. (1999) Development of quality of life questionnaire in Japan: quality of life assessment of cancer patients receiving chemotherapy. Psychooncology 8: 355-363

9. Proctor MJ, Morrison DS, Talwar D, et al. (2011) An inflammationbased prognostic score (mGPS) predicts cancer survival independent of tumour site: a Glasgow Inflammation Outcome Study. Br J Cancer 104:726-734

10. Kim KH (2005) The relation among fit indexes, power, and sample size in structural equation modeling. Struct Equ Model 12:368-390

11. Fearon K, Strasser F, Anker SD, et al. (2011) Definition and classification of cancer cachexia: an international consensus. Lancet Oncol 12:489-495

12. Temel JS, Greer JA, Muzikansky A, et al. (2010) Early palliative care for patients with metastatic non-small-cell lung cancer. N Engl J Med 363:733-742

13. Aapro M, Arends J, Bozzetti F, et al. (2014) Early recognition of malnutrition and cachexia in the cancer patient: a position paper of a European School of Oncology Task Force. Ann Oncol 25: 1492-1499

14. Prado CM, Baracos VE, McCargar LJ, et al. (2007) Body composition as an independent determinant of 5fluorouracil-based chemotherapy toxicity. Clin Cancer Res $13: 3264-3268$

15. Prado CM, Baracos VE, McCargar LJ, et al. (2009) Sarcopenia as a determinant of chemotherapy toxicity and time to tumor progression in metastatic breast cancer patients receiving capecitabine treatment. Clin Cancer Res 15:2920-2926

16. Stene GB, Helbostad JL, Balstad TR, et al. (2013) Effect of physical exercise on muscle mass and strength in cancer patients during treatment-a systematic review. Crit Rev Oncol Hematol 88:573-593

17. Garcia JM, Boccia RV, Graham CD, et al. (2015) Anamorelin for patients with cancer cachexia: an integrated analysis of two phase 2, randomized, placebo-controlled, double-blind trials. Lancet Oncol 16:108-116

18. Temel JS, Currow DC, Fearon K et al (2015) Phase III trials of anamorelin in patients with advanced non-small cell lung cancer (NSCLC) and cachexia (ROMANA 1 and 2). Abstract 9500. 2015 ASCO Annual Meeting, San Francisco, CA, August 7-8, 2015. J Clin Oncol 33 (suppl; abstr 9500) 